\title{
A Herschel PACS and SPIRE study of the dust content of the Cassiopeia A supernova remnant ${ }^{\star, \star \star}$
}

\author{
M. J. Barlow ${ }^{1}$, O. Krause ${ }^{2}$, B. M. Swinyard ${ }^{3}$, B. Sibthorpe ${ }^{4}$, M.-A. Besel ${ }^{2}$, R. Wesson ${ }^{1}$, R. J. Ivison ${ }^{4}$, L. Dunne ${ }^{5}$ \\ W. K. Gear ${ }^{6}$, H. L. Gomez ${ }^{6}$, P. C. Hargrave ${ }^{6}$, Th. Henning ${ }^{2}$, S. J. Leeks ${ }^{3}$, \\ T. L. Lim $^{3}$, G. Olofsson ${ }^{7}$, and E. T. Polehampton 3,8 \\ 1 Department of Physics and Astronomy, University College London, Gower Street, London WC1E 6BT, UK \\ e-mail: mjb@star.ucl.ac.uk \\ 2 Max-Planck-Institut für Astronomie, Königstuhl 17, 69117 Heidelberg, Germany \\ 3 Space Science and Technology Department, Rutherford Appleton Laboratory, Oxfordshire, OX11 0QX, UK \\ 4 UK Astronomy Technology Centre, Royal Observatory Edinburgh, Blackford Hill, Edinburgh EH9 3HJ, UK \\ 5 School of Physics and Astronomy, University of Nottingham, University Park, Nottingham NG7 2RD, UK \\ 6 School of Physics and Astronomy, Cardiff University, The Parade, Cardiff, Wales CF24 3AA, UK \\ 7 Dept of Astronomy, Stockholm University, AlbaNova University Center, Roslagstulsbacken 21, 10691 Stockholm, Sweden \\ 8 Institute for Space Imaging Science, University of Lethbridge, Lethbridge, Alberta, TJ1 1B1, Canada
}

Received 30 March 2010 / Accepted 12 May 2010

\section{ABSTRACT}

\begin{abstract}
Using the 3.5-m Herschel Space Observatory, imaging photometry of Cas A has been obtained in six bands between 70 and $500 \mu \mathrm{m}$ with the PACS and SPIRE instruments, with angular resolutions ranging from 6 to 37". In the outer regions of the remnant the 70- $\mu \mathrm{m}$ PACS image resembles the 24- $\mu \mathrm{m}$ image Spitzer image, with the emission attributed to the same warm dust component, located in the reverse shock region. At longer wavelengths, the three SPIRE bands are increasingly dominated by emission from cold interstellar dust knots and filaments, particularly across the central, western and southern parts of the remnant. Nonthermal emission from the northern part of the remnant becomes prominent at $500 \mu \mathrm{m}$. We have estimated and subtracted the contributions from the nonthermal, warm dust and cold interstellar dust components. We confirm and resolve for the first time a cool ( $35 \mathrm{~K})$ dust component, emitting at $70-160 \mu \mathrm{m}$, that is located interior to the reverse shock region, with an estimated mass of $0.075 M_{\odot}$.
\end{abstract}

Key words. ISM: supernova remnants - dust, extinction - Infrared: ISM

\section{Introduction}

The large quantities of dust found in many high-redshift sources (e.g. Priddey et al. 2003; Bertoldi et al. 2003) have often been interpreted as having originated in the ejecta of core-collapse supernovae (CCSNe) from massive stars. Models for CCSNe have predicted the formation of up to $0.1-1 M \odot$ of dust in their ejecta (e.g. Kozasa et al. 1991; Todini \& Ferrara 2001), which could be sufficient to account for the dust observed at high redshifts (Morgan \& Edmunds 2003; Dwek et al. 2007) and might provide a significant source of dust in the local Universe.

Cassiopeia A (Cas A), with an age of 330-340 years (Fesen et al. 2006) and a distance of $3.4 \mathrm{kpc}$ (Reed et al. 1995), is the youngest known core-collapse SNR in the Milky Way, so the mass of swept-up interstellar material is much less than that in the ejecta. From optical spectra of distant light echoes, Krause et al. (2008) identified it as the product of a hydrogen-deficient Type IIb CCSN. Cas A has been intensively studied by ISO and Spitzer at infrared wavelengths (e.g. Lagage et al. 1996; Tuffs et al. 1999; Arendt et al. 1999; Douvion et al. 2001; Hines et al. 2004; Ennis et al. 2006; Rho et al. 2008; Smith et al. 2009).

\footnotetext{
* Herschel is an ESA space observatory with science instruments provided by European-led Principal Investigator consortia and with important participation from NASA.

$\star \star$ Figure 3 is only available in electronic form at http://www . aanda.org
}

Arendt et al. (1999) derived $0.038 M_{\odot}$ of $52 \mathrm{~K}$ dust from a fit to the IRAS 60- and 100- $\mu$ m fluxes, while Rho et al. (2008) estimated $0.020-0.054 M_{\odot}$ of $65-265 \mathrm{~K}$ dust to be emitting between 5 and $70 \mu \mathrm{m}$, particularly in a bright ring coincident with the reverse shock. From 450- and 850- $\mu \mathrm{m}$ SCUBA observations, Dunne et al. (2003) reported the presence of excess emission over nonthermal flux levels extrapolated from the radio, which they attributed to 2-4 $M_{\odot}$ of "cold" ( $\left.T \sim 15-20 \mathrm{~K}\right)$ dust. However, Krause et al. (2004) argued that most of the submm excess emission could be due to dust in foreground molecular clouds and derived an upper limit of $0.2 M_{\odot}$ for cold dust within the remnant. Dunne et al. (2009) reported that the 850- $\mu \mathrm{m}$ emission from Cas A was polarized at a significantly higher level than its radio synchrotron emission and attributed this to $\sim 1 M_{\odot}$ of cold dust or alternatively a significantly smaller quantity of iron needles. Iron needles were originally proposed by Dwek (2004) and produce a very different SED to "traditional grains", with very little flux present at $\lambda<500 \mu \mathrm{m}$. Such grains would be consistent with a high polarised fraction.

Nozawa et al. (2010) modelled the evolution of dust in Cas A and found that the observed infrared SED of Cas A is reproduced by $0.08 M_{\odot}$ of newly formed dust, $0.072 M_{\odot}$ of which they inferred to consist of $\sim 40-\mathrm{K}$ dust in the unshocked regions inside the reverse shock. This is supported by recent AKARI and BLAST 65-500- $\mu$ m photometric observations of Cas A reported by Sibthorpe et al. (2010). Although they concluded that at their 

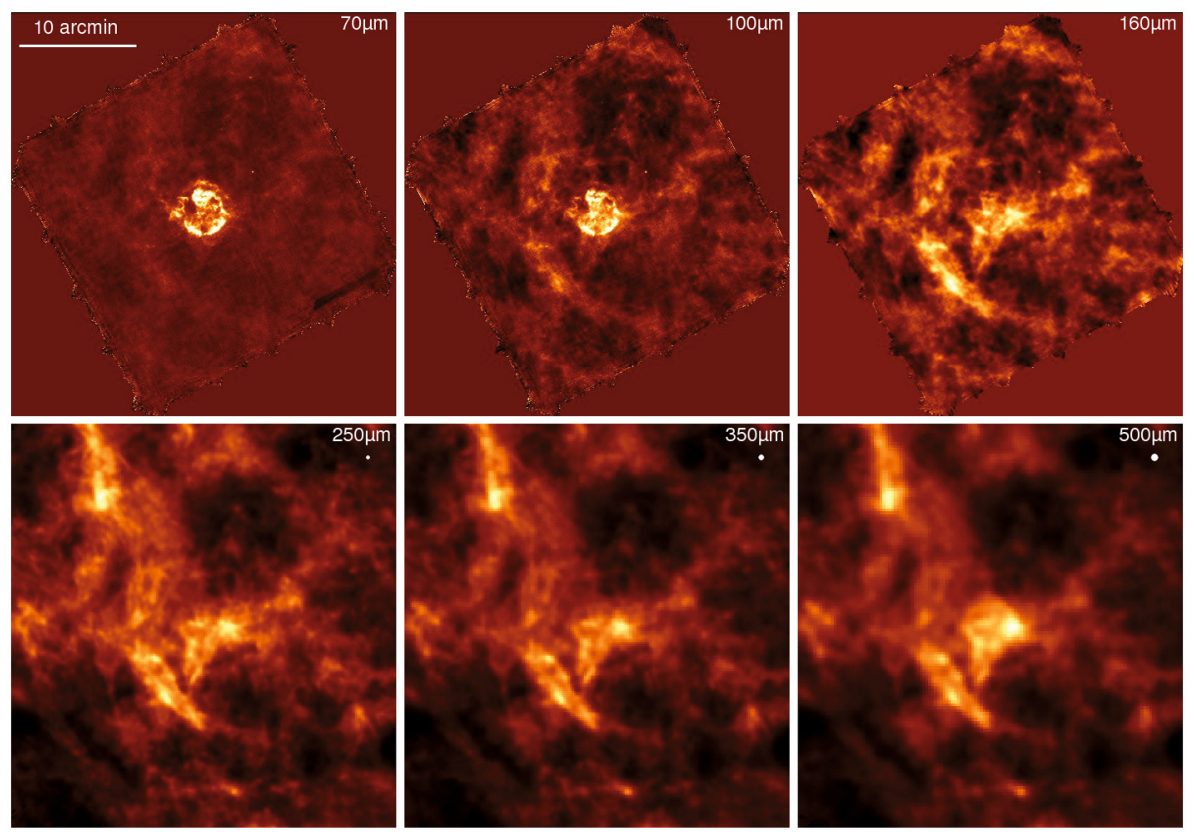

Fig. 1. Images of Cas A, obtained in the three PACS bands (top row) and in the three SPIRE bands (bottom row), centred at $23 \mathrm{~h} 23 \mathrm{~m} 26.3 \mathrm{~s}$ $+58^{\circ} 48^{\prime} 51.33^{\prime \prime}(\mathrm{J} 2000.0)$. North is up and east is to the left. The full width half maximum $(F W H M)$ angular resolutions of the SPIRE images, indicated by the filled white circles at the top-right of each SPIRE image, are respectively 18,25 and $37^{\prime \prime}$ at 250,350 and $500 \mu \mathrm{m}$. With the PACS scan-map speed of $20^{\prime \prime} / \mathrm{s}$ the $F W H M$ resolutions were 5.8, 7.8 and $12.0^{\prime \prime}$ at 70, 100 and $160 \mu \mathrm{m}$, respectively. longest wavelengths they could not isolate any cold dust emission from the SNR from confusing interstellar emission, they did however find evidence for a $\sim 33-\mathrm{K}$ "cool" dust component, peaking at about $100 \mu \mathrm{m}$, with an estimated mass of $\sim 0.06 M_{\odot}$. In the present paper we present new far-IR and submm observations of Cas A obtained with the Herschel Space Observatory (Pilbratt et al. 2010).

\section{Observations}

Cas A was observed with the SPIRE imaging photometer on 2009 Sep. 12 and Dec. 17. The SPIRE instrument and its inorbit performance are described by Griffin et al. (2010), and the SPIRE astronomical calibration methods and accuracy are outlined by Swinyard et al. (2010). The photometer's absolute flux calibration uncertainty is estimated to be $15 \%$. On each occasion scan maps covering a $32^{\prime} \times 32^{\prime}$ area centred on Cas A were obtained simultaneously at 250,350 and $500 \mu \mathrm{m}$, with an on-source integration time of $2876 \mathrm{~s}$. The remnant was observed with the PACS imaging photometer on 2009 Dec. 17. The PACS instrument, its in-orbit performance and calibration are described by Poglitsch et al. (2010); the absolute flux calibration uncertainty of the photometer is estimated to be $20 \%$. Scan maps comprised of two orthogonal scan legs, each of $22^{\prime}$ in length, were obtained using the $70+160-\mu \mathrm{m}$ and $100+160-\mu \mathrm{m}$ channels. For each pair of filters the on-source integration time was $2376 \mathrm{~s}$.

A montage showing the images in the six PACS and SPIRE bands is presented in Fig. 1. The PACS $70-\mu \mathrm{m}$ image (Fig. 1, top-left and Fig. 2, top-right) strongly resembles the similar angular resolution Spitzer 24- $\mu$ m MIPS image (Hines et al. 2004); a bright ring of warm dust emission is coincident with the reverse shock, while the fainter outer emission edge coincides with the forward shock. In the longer wavelength images, knots and lanes of diffuse interstellar dust emission envelope the SNR this emission is particularly bright at the central, western and southern parts of the remnant, where its morphology closely matches that of molecular line maps, such as the ${ }^{13} \mathrm{CO}$ emission map presented by Wilson \& Batrla (2005). In the SPIRE 500- $\mu \mathrm{m}$ image, the nonthermal emission from the northern parts of the remnant becomes prominent, coincident with emission seen with SCUBA at $850 \mu \mathrm{m}$ (Dunne et al. 2003).
Table 1. Total and individual component flux densities (in Jy) for Cas A.

\begin{tabular}{lcccccc}
\hline \hline & $70 \mu \mathrm{m}$ & $100 \mu \mathrm{m}$ & $160 \mu \mathrm{m}$ & $250 \mu \mathrm{m}$ & $350 \mu \mathrm{m}$ & $500 \mu \mathrm{m}$ \\
\hline Published & $107^{1}$ & $105^{2}$ & $101^{2}$ & $76^{3}$ & $49^{3}$ & $42^{3}, 70^{4}$ \\
& \pm 22 & \pm 21 & \pm 20 & \pm 16 & \pm 10 & $\pm 8, \pm 16$ \\
Herschel & 169 & 192 & 166 & 168 & 92 & 52 \\
& \pm 17 & \pm 19 & \pm 17 & \pm 17 & \pm 10 & \pm 7 \\
Nonthermal & 6.3 & 8.1 & 11.2 & 15.4 & 19.4 & 24.9 \\
& \pm 0.6 & \pm 0.7 & \pm 0.9 & \pm 1.1 & \pm 1.3 & \pm 1.6 \\
Warm dust & 120 & 63 & 22 & 7.0 & 3.1 & 1.2 \\
& \pm 12 & \pm 6 & \pm 2 & \pm 0.8 & \pm 0.4 & \pm 0.2 \\
Cold IS dust & 18 & 76 & 123 & 141 & 69 & 27.5 \\
& \pm 4 & \pm 11 & \pm 17 & \pm 17 & \pm 10 & \pm 7 \\
Cool dust & 25 & $29^{5}$ & 10 & 4.6 & 0.5 & -1.6 \\
& \pm 7 & \pm 11 & \pm 17 & \pm 17 & \pm 10 & \pm 4 \\
\hline
\end{tabular}

Notes. (1) MIPS (Hines et al. 2004); (2) AKARI 90 and $170 \mu \mathrm{m}$ (Sibthorpe et al. 2010); (3) BLAST 250, 350 and $500 \mu \mathrm{m}$ (Sibthorpe et al. 2010); ${ }^{(4)}$ SCUBA $450 \mu \mathrm{m}$ (Dunne et al. 2003); ${ }^{(5)}$ after subtracting a line contribution of $16 \mathrm{Jy}$ (see Sect. 3).

The second row of Table 1 lists the total flux density measured from Cas A in each of the six Herschel bands, using a $165^{\prime \prime}$ radius aperture that should encompass everything within the forward shock region, located at $153 \pm 12^{\prime \prime}$ (Gotthelf et al. 2001). These total flux densities were measured relative to four "floor" regions located to the north and southwest of the nebula. The total flux densities listed for Cas A include the emission from the cold interstellar dust that is superposed on the remnant. The first row of Table 1 lists previously published flux densities for Cas A at wavelengths in common. The SPIRE 500- $\mu$ m flux overlaps the SCUBA and BLAST $450 / 500-\mu$ m fluxes (Dunne et al. 2003; Sibthorpe et al. 2010) but at shorter wavelengths the PACS and SPIRE flux densities are factors of 1.6-2.2 times larger than published values from IRAS and Spitzer (Hines et al. 2004) and from AKARI and BLAST (Sibthorpe et al. 2010) that are listed in the first row of Table 1 . We attribute these differences to the fact that the higher angular resolution of Herschel enabled lower "floor" points in the diffuse background emission to be resolved and subtracted, whereas the lower angular resolutions of smaller aperture telescopes, at wavelengths in common, 



Fig. 2. Images of Cas A at infrared, submillimetre and radio wavelengths. The top six images are $7^{\prime}$ on a side, while the lower three images are $10^{\prime}$ on a side, with inset boxes showing the $7^{\prime}$ field. North is up and east is to the left. The inner and outer circles in the middle-right image respectively show the positions of the reverse and forward shocks according to Gotthelf et al. (2001), while the $165^{\prime \prime}$-radius circle in the top-middle image encloses the area for which the fluxes listed in Table 1 were measured. See text for further details.

is likely to have caused higher mean background levels to be estimated.

\section{Emission component decomposition}

In order to investigate the "cool" dust emission component in Cas A that was diagnosed by Sibthorpe et al. (2010) from an analysis of their AKARI and BLAST data, we have followed a similar procedure by attempting to identify and subtract the contributions made at each wavelength by (a) the remnant's nonthermal (synchrotron) emission; (b) the warm dust component that dominates the Spitzer 24- $\mu$ m image; and (c) the cold interstellar dust component. In addition, we have estimated the contributions made by line emission to the PACS in-band fluxes.

The nonthermal component: we extracted from the archive and reprocessed a 6-cm VLA dataset on Cas A obtained in 1997/8, convolving it to $6 "$ resolution, as shown in Fig. 2 (upper left). Also shown in Fig. 2 is the 3.6- $\mu$ m IRAC image obtained by Ennis et al. (2006), which was also convolved to a resolution of $6^{\prime \prime}$; as Ennis et al. noted, the morphologies of the 6-cm and $3.6-\mu \mathrm{m}$ images correspond very closely, indicating that both are dominated by the nonthermal emission component. We corrected the 3.6- $\mu \mathrm{m}$ image for extinction based on the X-ray absorption results of Willingale et al. (2002) and ratioed the two nonthermal images to produce a spectral index map which was quite smooth, yielding a mean spectral index of $-0.70 \pm 0.05$. We therefore adopted a spectral index of -0.70 to estimate the remnant's nonthermal emission in each of the six PACS and SPIRE bands (third row of Table 1) and to generate images convolved to the resolution of each of the bands. The other images in Fig. 2 have had the appropriate nonthermal component image subtracted.
The warm dust component: Figure 2 (middle-top panel) shows the 24- $\mu \mathrm{m}$ MIPS image obtained by Hines et al. (2004). They noted the similarity between the 24- and 70- $\mu \mathrm{m}$ MIPS images, pointing to a common emitting component, which we term the "warm dust" component, peaking in a bright ring coincident with the position of the reverse shock. Our PACS $70-\mu \mathrm{m}$ image (Fig. 2, central panel) has a similar resolution to the MIPS $24-\mu \mathrm{m}$ image and shows a similar outer morphology, but more emission is evident from the remnant's interior in the $70-\mu \mathrm{m}$ image. We therefore normalised the MIPS $24-\mu \mathrm{m}$ image to the surface brightness levels in the outer parts of the remnant in the PACS 70- $\mu \mathrm{m}$ image and subtracted it, to obtain the difference image shown in the middle-right panel of Fig. 2. The total "warm dust" contribution at $70 \mu \mathrm{m}$, obtained from the scaled-up $24-\mu \mathrm{m}$ image, is $120 \pm 6 \mathrm{Jy}$. We extrapolated this warm dust component from $70 \mu \mathrm{m}$ to longer wavelengths using the predicted spectrum from $3 \times 10^{-3} M_{\odot}$ of $82-\mathrm{K}$ magnesium protosilicate, found by Hines et al. (2004) to fit the $24-70-\mu \mathrm{m}$ MIPS spectrum, in order to obtain the flux densities listed in the 4th row of Table 1. Warm dust component images, convolved to the appropriate angular resolutions, were subtracted from the images obtained at $100 \mu \mathrm{m}$ and longwards, as were the appropriate nonthermal images, before estimating and subtracting the contribution from superposed cold interstellar dust, discussed next.

The cold interstellar and cool Cas A dust components: The bottom row of Fig. 2 shows the 160, 350 and 500- $\mu$ m images of Cas A after subtracting scaled images of the other components. For illustration purposes they are shown convolved to the same $37^{\prime \prime}$ resolution as the $500-\mu \mathrm{m}$ image. These residual images show a strikingly similar morphology, indicating that they are emitted by the same cold interstellar dust particles. To obtain these maps in an iterative way we started with maps corrected 
for the nonthermal and warm components and determined average $100 / 160$ and $70 / 160-\mu \mathrm{m}$ flux ratios for several bright regions located outside the remnant. We then applied these ratios to the 160- $\mu \mathrm{m}$ image and subtracted them from 70- and 100$\mu \mathrm{m}$ images which had been convolved to the $160-\mu \mathrm{m}$ resolution. A consistent cool dust morphology is seen in the resulting 70 and $100-\mu \mathrm{m}$ "cool dust" images (Fig. 2, middle-right and topright). Note that in this first step, the $160-\mu \mathrm{m}$ map initially still contained a contribution from the cool SN dust component. In order to determine its contribution at $160 \mu \mathrm{m}$, we subtracted a scaled image of the cool component at $70 \mu \mathrm{m}$ (where the ISM and line contamination is smallest) from the $160-\mu \mathrm{m}$ map iteratively, until its visible imprint was minimized. This corrected $160-\mu \mathrm{m}$ ISM map was then used iteratively to obtain more accurate 70 - and $100-\mu \mathrm{m}$ images of the cool dust component. Our estimates for the flux densities in each band from the cold interstellar dust emission that is superposed on the remnant are listed in the penultimate row of Table 1 . We note that the relative uncertainties of individual emission components are smaller than the absolute calibration uncertainties associated with the total flux densities.

Emission line contributions to the PACS bands: archival ISOLWS 43-197- $\mu$ m grating spectra, obtained with an aperture size of $80^{\prime \prime}$, exist for six positions across Cas A, and for one offset position (see Fig. 4 of Docenko \& Sunyaev 2010). The spectra show strong broad emission from the [O I] $63-\mu \mathrm{m}$ and [O III] $52-$ and $88-\mu \mathrm{m}$ lines. After convolving with the filter and instrumental response functions, the line contribution to the 70- and $160-\mu \mathrm{m}$ bands was found to be negligible but the $88-\mu \mathrm{m}$ line was found to make a $\sim 16 \mathrm{Jy}$ contribution to the PACS $100-\mu \mathrm{m}$ band - this has been subtracted to give the 100- $\mu \mathrm{m}$ "cool dust" flux density listed in the last row of Table 1. The spectral energy distributions of each of the emitting components are plotted in Fig. 3 in the online.

\section{Discussion: the mass of cool dust in Cas $A$}

Following subtraction of the nonthermal, warm dust and cold interstellar dust components, the 100- $\mu \mathrm{m}$ image shown in Fig. 2 (top-right) shows a similar morphology to the cool dust $70-\mu \mathrm{m}$ image shown below it. These represent the first resolved images of this dust component, whose existence was also inferred by Tuffs et al. (2005; 60-200- $\mu$ m ISOPHOT) and Sibthorpe et al. (2010; 65-500- $\mu \mathrm{m}$ AKARI/BLAST). The flux densities in each band from the cool dust component are listed in the final row of Table 1. They can be fitted (Fig. 3) by $0.075 \pm 0.028 M_{\odot}$ of $35 \pm 3-\mathrm{K} \lambda^{-2}$ emissivity silicate dust having a $160-\mu \mathrm{m}$ absorption coefficient of $9.8 \mathrm{~cm}^{2} \mathrm{~g}^{-1}$ (Dorschner et al. 1995). Sibthorpe et al. derived a $33-\mathrm{K}$ cool dust mass of $0.055 M_{\odot}\left(0.066 M_{\odot}\right.$ with the dust absorption coefficients used here), consistent with our own estimate.

Nozawa et al. (2010) modelled the Hines et al. (2004) 8-100- $\mu \mathrm{m}$ SED of Cas A with $0.008 M_{\odot}$ of shock-heated warm dust and $0.072 M_{\odot}$ of unshocked cool dust in the remnant's interior. Their dust formation model for the Cas A ejecta predicted $0.17 M_{\odot}$ of new dust, from which they suggested that $0.09 M_{\odot}$ had already been destroyed by the reverse shock. If the $0.075 M_{\odot}$ of cool interior dust that we find here is to survive its $5000 \mathrm{~km} \mathrm{~s}^{-1}$ encounter with the reverse shock, it will need to be protected by being inside very dense clumps. If most of the dust was eventually destroyed, then remnants of this type would not make a significant contribution to the dust content of the ISM, and could even dilute it.
The present observations provide no direct evidence for the presence of significant quantities of cold $(<25 \mathrm{~K})$ dust within Cas $\mathrm{A}$ - the $500-\mu \mathrm{m}$ emission that is visible from the least obscured, northern, part of the remnant is, to first order, removed when the predicted nonthermal emission contribution is subtracted (Fig. 2; bottom-right). The cause of the 850- $\mu$ m excess in the SCUBA map of the northern part of the remnant is therefore unresolved. Spectroscopic observations are planned with PACS and SPIRE for various on- and off-remnant positions. These should enable a clean separation of line contributions and a full sampling of the continuum energy distribution from 50-670 $\mu \mathrm{m}$.

Acknowledgements. PACS has been developed by a consortium of institutes led by MPE (Germany) and including UVIE (Austria); KUL, CSL, IMEC (Belgium); CEA, OAMP (France); MPIA (Germany); IFSI, OAP/AOT, OAA/CAISMI, LENS, SISSA (Italy); IAC (Spain). This development has been supported by the funding agencies BMVIT (Austria), ESA-PRODEX (Belgium), CEA/CNES (France), DLR (Germany), ASI (Italy), and CICT/MCT (Spain). SPIRE has been developed by a consortium of institutes led by Cardiff Univ. (UK) and including Univ. Lethbridge (Canada); NAOC (China); CEA, LAM (France); IFSI, Univ. Padua (Italy); IAC (Spain); Stockholm Observatory (Sweden); Imperial College London, RAL, UCL-MSSL, UKATC, Univ. Sussex (UK); Caltech, JPL, NHSC, Univ. Colorado (USA). This development has been supported by national funding agencies: CSA (Canada); NAOC (China); CEA, CNES, CNRS (France); ASI (Italy); MCINN (Spain); SNSB (Sweden); STFC (UK); and NASA (USA). These observations were taken as part of the Science Demonstration Phase of the Mass-loss of Evolved StarS (MESS) Guaranteed Time Key Programme. (Groenewegen et al., in prep.)

\section{References}

Arendt, R. G., Dwek, E., \& Moseley, S. H. 1999, ApJ, 521, 234 Bertoldi, F., Carilli, C. L., Cox, P., et al. 2003, A\&A, 406, L55 Docenko, D., \& Sunyaev, R. A. 2010, A\&A, 509, A59 Dorschner, J., Begemann, B., Henning, T., et al. 1995, A\&A, 300, 503 Douvion, T., Lagage, P. O., \& Cesarsky, C. J. 1999, A\&, 352, L111 Dunne, L., Eales, S., Ivison, R., et al. 2003, Nature, 424, 285 Dunne, L., Maddox, S. J., Ivison, R. J., et al. 2009, MNRAS, 394, 1307 Dwek, E. 2004, ApJ, 607, 848

Dwek, E., Arendt, R. G., Fixsen, D. J., et al. 1997, ApJ, 475, 565 Dwek, E., Galliano, F., \& Jones, A. P. 2007, ApJ, 662, 927

Ennis, J. A., Rudnick, L., Reach, W. T., et al. 2006, ApJ, 652, 376 Fesen, R. A., Hammell, M. C., Morse, J., et al. 2006, ApJ, 645, 283 Gotthelf, E. V., Koralesky, B., Rudnick, L., et al. 2001, ApJ, 552, L39 Griffin, M. J., Abergel, A., Abreu, A., et al. 2010, A\&A, 518, L3 Hines, D. C., Rieke, G. H., Gordon, K. D., et al. 2004, ApJS, 154, 290 Kozasa, T., Hasegawa, H., \& Nomoto, K. 1991, A\&A 249, 474 Krause, O., Birkmann, S. M., Rieke, G. H., et al. 2004, Nature, 432, 596 Krause, O., Birkmann, S. M., Usuda, T., et al. 2008, Science, 320, 1195 Lagage, P. O., Claret, A., Ballet, J., et al. 1996, A\&A, 315, L273 Morgan, H. L., \& Edmunds, M. G. 2003, MNRAS, 343, 427 Nozawa, T., Kozasa, T., Tominaga, N., et al. 2010, ApJ, 713, 356 Pilbratt, G. L., Riedinger, J. R., Passvogel, T., et al. 2010, A\&A, 518, L1 Poglitsch, A., Waelkens, C., Geis, N., et al. 2010, A\&A, 518, L2 Priddey, R. S., Isaak, K. G., McMahon, R. G., Robson, E. I., \& Pearson, C. P. 2003, MNRAS, 344, L74

Reed, J. E., Hester, J. J., Fabian, A. C., \& Winkler, P. F. 1995, ApJ, 440, 706 Rho, J., Kozasa, T., Reach, W. T., et al. 2008, ApJ, 673, 271

Sibthorpe, B., Ade, P. A. R., Bock, J. J., et al. 2010, ApJ, accepted [arXiv: 0910.1094]

Smith, J. D. T., Rudnick, L., Delaney, T., et al. 2009, ApJ, 693, 713

Swinyard, B. M., Ade, P., Baluteau, J. P., et al. 2010, A\&A, 518, L4 Todini, P., \& Ferrara, A. 2001, MNRAS 325, 726

Tuffs, R. J., Fischera, J., Drury, L. O’C., et al. 1999, in The Universe as seen by ISO, ed. P. Cox, \& M. Kessler, ESA-SP, 427, 241

Tuffs, R. J., Popescu, C. C., \& Völk, H. J. 2005, in The Dusty and Molecular Universe, ed. A. Wilson, ESA-SP, 577, 427

Willingale, R., Bleeker, J. A. M., van der Heyden, K. J. \& Kaastra, J. S. 2002, A\&A, 381, 1039

Wilson, T. L., \& Batrla, W. 2005, A\&A, 430, 561

Page 5 is available in the electronic edition of the journal at http://www . aanda. org 
M. J. Barlow et al.: A Herschel PACS and SPIRE study of the dust content of the Cassiopeia A supernova remnant

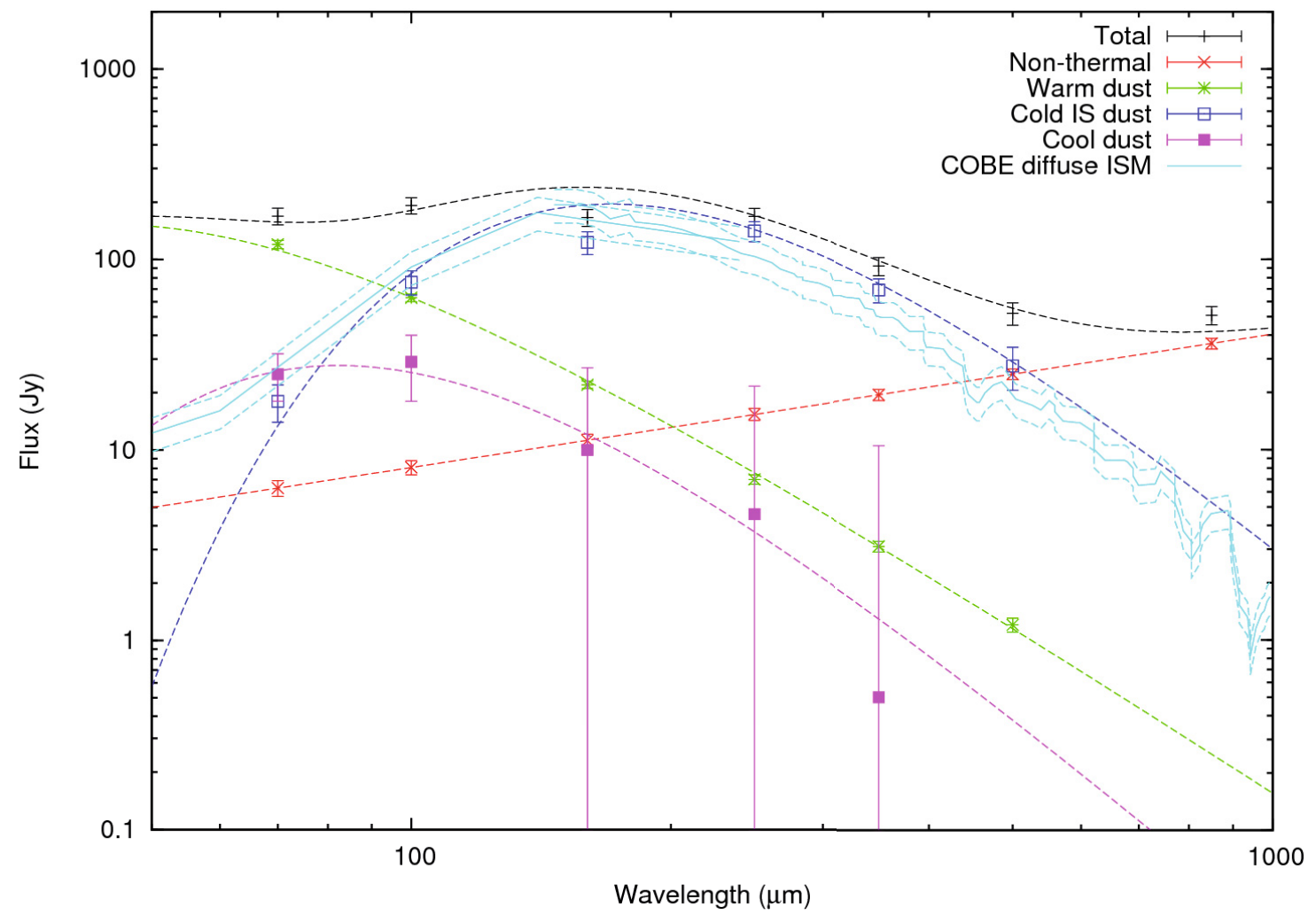

Fig. 3. The derived 70-850- $\mu \mathrm{m}$ spectral energy distributions of the components contributing to the observed emission from Cas A. See Sect. 3 for a description of how the flux densities from each component at each wavelength were estimated. Red: nonthermal flux densities estimated from a power-law fit between the 6-cm and 3.6- $\mu \mathrm{m}$ flux densities; Green: warm dust component flux densities; Blue: flux densities for the cold interstellar dust component. Also shown are a $\lambda^{-2}$ emissivity $17-\mathrm{K}$ fit to the cold IS dust flux densities (blue dashed line), and a comparison with the Dwek et al. (1997) COBE DIRBE/FIRAS average ISM spectral energy distribution (turquoise; shown with the quoted $\pm 20 \% 1 \sigma$ uncertainty limits). Magenta: the derived flux densities for the cool dust component, together with a $35-\mathrm{K} \lambda^{-2}$ emissivity fit (dashed magenta line). The blue points show the PACS and SPIRE 70-500- $\mu$ m total flux densities measured for Cas A, as well as the SCUBA 850- $\mu$ m flux density measured by Dunne et al. (2003). The black dashed line corresponds to the sum of the fits to the nonthermal, warm dust, cool dust and cold IS dust components. 